\section{Prevalence and characteristics of migraine in CADASIL}

Cephalalgia

2016, Vol. 36(II) 1038-1047

(C) International Headache Society 2015 Reprints and permissions: sagepub.co.uk/journalsPermissions.nav DOI: $10.1177 / 0333102415620909$ cep.sagepub.com

@AGE

\author{
Stephanie Guey ${ }^{1,2}$, Jérôme Mawet ${ }^{1,3}$, Dominique Hervé ${ }^{1,2}$, \\ Marco Duering ${ }^{4}$, Ophelia Godin', Eric Jouvent ${ }^{1,2}$, \\ Christian Opherk ${ }^{5}$, Nassira Alili', Martin Dichgans ${ }^{4,6}$ \\ and Hugues Chabriat ${ }^{1,2}$
}

\begin{abstract}
Background and objective: Migraine with aura (MA) is a major symptom of cerebral autosomal dominant arteriopathy with subcortical infarcts and leukoencephalopathy (CADASIL). We assessed the spectrum of migraine symptoms and their potential correlates in a large prospective cohort of CADASIL individuals.

Methods: A standardized questionnaire was used in 378 CADASIL patients for assessing headache symptoms, trigger factors, age at first attack, frequency of attacks and associated symptoms. MRI lesions and brain atrophy were quantified. Results: A total of $54.5 \%$ of individuals had a history of migraine, mostly MA in $84 \%$ of them; $62.4 \%$ of individuals with MA were women and age at onset of MA was lower in women than in men. Atypical aura symptoms were experienced by $59.3 \%$ of individuals with MA, and for $19.7 \%$ of patients with MA the aura was never accompanied by headache. MA was the inaugural manifestation in $41 \%$ of symptomatic patients and an isolated symptom in $12.1 \%$ of individuals. Slightly higher MMSE and MDRS scores and lower Rankin score were detected in the MA group.

Conclusion: MA is observed in almost half of all CADASIL patients. Atypical aura symptoms are reported by more than one in two of them. MA is often inaugural, can remain isolated and is not associated with the severity of the disorder.
\end{abstract}

\title{
Keywords
}

CADASIL, migraine, aura, cortical spreading depression, small vessel disease

Date received: 2 July 20I5; revised: 2 October 20I5; 2 November 20I5; accepted: 5 November 2015

\section{Introduction}

Cerebral autosomal dominant arteriopathy with subcortical infarcts and leukoencephalopathy (CADASIL) is the most frequent hereditary ischemic small vessel disease of the brain (1). Mutations in the extracellular domains of the NOTCH3 receptor responsible for the disease may favor oligomerization of the protein and its accumulation in the wall of arterioles and capillaries (2). CADASIL is responsible for recurrent ischemic stroke during mid-adulthood and can lead to severe motor disability and cognitive decline (3-5).

Migraine with aura (MA) is a major symptom of CADASIL. A positive history of MA has been previously reported in $20 \%$ to $40 \%$ of symptomatic individuals. MA can occur several decades before stroke events. In contrast to MA, migraine without aura (MO) was not found to be more prevalent in CADASIL than in different large population-based samples $(3,4,6-8)$. Mean age at onset of MA was previously found to be close to 30 years in CADASIL (9). In a retrospective study of 41 CADASIL patients with

'Department of Neurology, GH Saint-Louis-Lariboisière, Assistance Publique des Hôpitaux de Paris APHP, Université Paris Denis Diderot and DHU NeuroVasc Sorbonne Paris-Cité, France

${ }^{2}$ INSERM, UMR II6I, France

${ }^{3}$ Emergency Headache Center, GH Saint-Louis-Lariboisière, Assistance Publique des Hôpitaux de Paris APHP, Université Paris Denis Diderot and DHU NeuroVasc Sorbonne Paris-Cité, France

${ }^{4}$ Institute for Stroke and Dementia Research, Klinikum der Universität

München, Ludwig-Maximilians-University, Germany

${ }^{5}$ Department of Neurology, SLK-Kliniken, Germany

${ }^{6}$ Munich Cluster for Systems Neurology (SyNergy), Germany

\section{Corresponding author:}

Hugues Chabriat, Department of Neurology, Hopital Lariboisière, 2 rue A Paré, 75010 Paris, France.

Email: hugues.chabriat@Irb.aphp.fr 
MA, Vahedi et al. reported that a large proportion of CADASIL patients experienced aura without headache or atypical aura such as hemiplegic or basilar aura, prolonged aura or acute-onset aura (9). Further analysis of the spectrum of MA symptoms and of their correlates is, however, hampered by the limited number of patients and retrospective nature of previous studies.

Herein, we aimed to analyze the frequency and clinical features of migraine symptoms with their clinical and imaging correlates in a large prospective cohort of 378 CADASIL individuals.

\section{Methods}

\section{Participants and clinical assessment}

Patients were recruited between 2003 and 2011 in a large prospective cohort study of CADASIL patients performed in Lariboisière Hospital, Paris, and in the Ludwig Maximilians University Neurology Clinic, Munich. All patients recruited in both centers during the inclusion period were included in this study. All patients carried a typical mutation of the NOTCH3 gene responsible for the disease or were diagnosed by the presence of pathognomonic granular osmiophilic deposits in skin biopsy $(1,2)$. They were aged 18 years or older at inclusion and gave their written consent to participate in the study. An independent ethics committee approved the study in both centers.

In each center, an experienced neurologist collected during an individual consultation a detailed history of headache symptoms as well as multiple clinical and demographic data such as age, sex, comorbidities, cardiovascular risk factors (arterial hypertension, diabetes, smoking and alcohol habits, dyslipidemia), history of stoke or transient ischemic attack (TIA), psychiatric symptoms, epilepsy, gait disturbances and cognitive impairment. Dementia was diagnosed according to the Diagnostic and Statistical Manual of Mental Disorders, fourth edition (DSM-IV) criteria.

Headache symptoms were classified according to the International Classification of Headache Disorders (10). A neurologist collected all information relative to headache history based on a standardized questionnaire. The following items were systematically collected: the type of migraine attacks (MO, MA), the most frequent triggering factors, the age at first attack, the mean frequency of attacks, the mean duration of headache during the two years prior to inclusion, the characteristics of headache (hemicrania, pulsatility) and the frequency of associated symptoms (nausea, vomiting, phonophobia, photophobia). For these different items, we pooled percentages of patients in categories as "often" (more than half of attacks) and "sometimes" (less than half of attacks). In patients reporting MA, the following items were also collected: the type of aura symptoms (visual, sensitive, motor, aphasic or other symptoms), the mean duration of aura, the occurrence of a typical "marche migraineuse" (progressive development of aura symptoms over $\geq 5$ minutes) and the chronological relationship of aura with headache. Aura without headache was considered as part of MA. Aura symptoms were considered to correspond to a typical aura when they were visual, sensory or aphasic, when they gradually developed over $\geq 5$ minutes and when their duration was between 5 and 60 minutes. Aura was considered as atypical when these characteristics were not present and corresponded to hemiplegic, basilar or ophthalmoplegic migraine; acute-onset aura (onset of symptoms in less than 5 minutes) or long-lasting aura (aura lasting more than an hour for only one symptom or more than four hours in presence of multiple symptoms). When the nature of a transient neurologic event was unclear, the episode was classified as indistinguishable between aura symptoms and a TIA.

All participants underwent a complete neurological examination including a global evaluation of the cognitive status with the Mini-Mental State Examination (MMSE) and the Mattis Dementia Rating scale (MDRS) and of the severity of disability with the modified Rankin scale (mRS).

\section{Imaging data}

Magnetic resonance imaging (MRI) was performed with a 1.5 Tesla system in both centers. The sequences and methods used for evaluating white matter hyperintensities (WMH), lacunes and microbleeds have been detailed elsewhere (11). The total volume of $\mathrm{WMH}$ was normalized to the intracranial cavity (ICC) in each patient $(\mathrm{nWMH}=$ [volume of $\mathrm{WMH} /$ volume ICC]*100). The total volume of lacunes in each patient was also normalized to the ICC (nLV $=$ [volume of lacunes/volume ICC]*100). Microbleeds were defined as rounded foci $\leq 5 \mathrm{~mm}$ in diameter hypointense on gradient-echo sequences. For obtaining brain volumes at baseline, the SIENAX algorithm was applied using T1-weighted images as previously reported (12). Normalized brain volumes corrected for different skull sizes (or brain parenchymal fraction $(\mathrm{BPF})=$ brain $\quad$ volume/intracranial cavity volume) were then used for statistical analyses. The volume of $\mathrm{WMH}$, the volume of lacunes and BPF are expressed as percentages of the ICC volume.

\section{Statistical analysis}

A descriptive analysis was performed for the main parameters obtained in the cohort. For each variable, 
percentages were calculated after excluding missing data. Imaging and clinical parameters were compared between patients with MA and the other patients using both univariate analysis without adjustment and multivariate analysis adjusting for age, gender and current cardiovascular risk factors (arterial hypertension, diabetes and hypercholesterolemia, tobacco use and alcohol use). The relationships between continuous and categorical variables were tested using the Student's $t$ test for univariate analysis and analysis of covariance for multivariate analysis. The relationships between qualitative variables were tested using the $\mathrm{Chi}^{2}$ test and Fisher's exact test when needed. The CochranMantel-Haenszel test was used for comparisons between qualitative variables after adjustment for age, gender, current cardiovascular risk factors and educational level. The threshold for statistical significance was $p<0.05$.

\section{Results}

A total of 378 CADASIL patients were included in the study: 249 in Paris and 129 in Munich. There were 167 men $(44.2 \%)$ and 211 women $(55.8 \%)$; their mean age was 51.3 years (range 23-79 years). Diagnosis was established by genetic testing for 358 patients and by skin biopsy for 20 patients.

\section{Main characteristics of the entire study population}

Among the 378 individuals, 206 (54.5\%) had a positive history of migraine attacks. There were 234 (61.9\%) who had had at least one cerebrovascular event (366 ischemic strokes, three hemorrhagic strokes and 108 transient neurologic episodes considered as TIA or as indistinguishable between TIA and aura). Previous or persisting psychiatric or mood disturbances were observed in 164 patients $(43.4 \%)$. A total of 181 patients $(47.9 \%)$ complained of memory or concentration difficulties and 43 suffered from dementia (11.4\%). Thirty-five patients $(9.3 \%)$ presented at least one epileptic seizure.

On MRI, all patients but one had WMH. The MRI results are detailed in the supplementary material. Only a single patient had a normal MRI. This patient was a 32-year-old man who reported a single episode of visual-sensory-aphasic aura without headache and had a normal clinical examination. He carried a typical mutation in the $\mathrm{NOTCH} 3$ gene.

\section{Global features of headache episodes in the study population}

A total of 206 were found to have a positive history of migraine attacks in the cohort (54.5\% of the cohort).
Table I. Main headache subtypes observed in the cohort.

\begin{tabular}{lll}
\hline Headache subtypes & $\begin{array}{l}\text { N patients } \\
\text { (\% whole } \\
\text { cohort) }\end{array}$ & $\begin{array}{l}\text { Women } \\
\text { (\% headache } \\
\text { group) }\end{array}$ \\
\hline $\begin{array}{c}\text { Migraine subtypes } \\
\text { - MA attacks (total) }\end{array}$ & $173(45.8 \%)$ & $108(62.4 \%)$ \\
- MA attacks only & $119(31.5 \%)$ & $71(59.7 \%)$ \\
- MA attacks + MO attacks & $54(14.3 \%)$ & $37(68.5 \%)$ \\
- MO attacks (total) & $87(23 \%)$ & $57(65.5 \%)$ \\
- MO attacks only & $33(8.7 \%)$ & $20(60.6 \%)$ \\
Others types & $85(22.5 \%)$ & $46(54.1 \%)$ \\
- Episodic tension type & $19(5 \%)$ & $9(47.4 \%)$ \\
- Chronic tension type & &
\end{tabular}

MA: migraine with aura; MO: migraine without aura.

The frequency of the various subtypes of migraine attacks in the cohort population is detailed in Table 1.

There were 173 individuals, representing $45.8 \%$ of the whole cohort or $84 \%$ of the migraine patients, who had a history of MA attacks. Among them, 54 patients also reported MO attacks.

Thirty-three patients $(8.7 \%$ of the whole cohort) reported MO attacks only.

In both groups, there was a majority of women: $62.4 \%$ of individuals with MA, and $60.6 \%$ of those with $\mathrm{MO}$ attacks only were women.

\section{Main characteristics of MO attacks in the study population}

Among the 87 patients with $\mathrm{MO}$ attacks (33 with MO attacks only and 54 patients with both MO and MA attacks), the first episode of MO occurred between 16 and 30 years of age in 42 individuals (48\%). The frequency of MO attacks over the past two years and according to age is shown in Figure 1. This frequency was largely variable from more than one attack per week to less than one every two years, but in most cases the frequency was low (less than one attack per month in $64.4 \%$ of MO patients). Other characteristics of MO attacks are detailed in the supplementary material.

\section{Main characteristics of MA attacks in the study population}

Among the 173 individuals with MA attacks, the peak age at onset of MA attacks was between 16 and 30 years in women and between 31 and 40 years in men (Figure 2). Among individuals with MA attacks, $54.2 \%$ of women had their first MA attack before they were 30 years old while $26.15 \%$ of men had their first attack before the same age limit $(p=0.0003)$. 


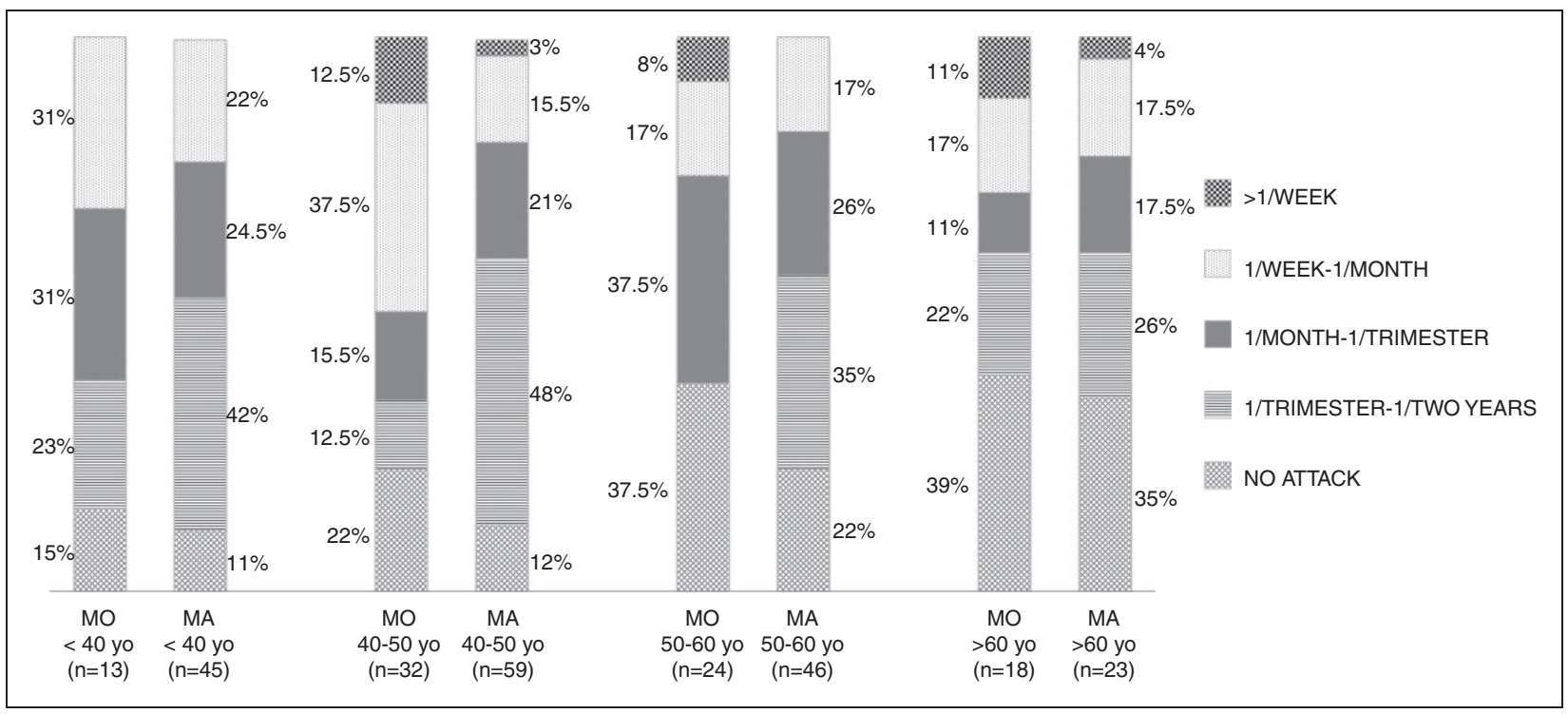

Figure I. Frequency of $M O$ and MA attacks over the past two years in CADASIL patients according to age.

MO: migraine without aura, MA: migraine with aura; CADASIL: cerebral autosomal dominant arteriopathy with subcortical infarcts and leukoencephalopathy; yo: years old.

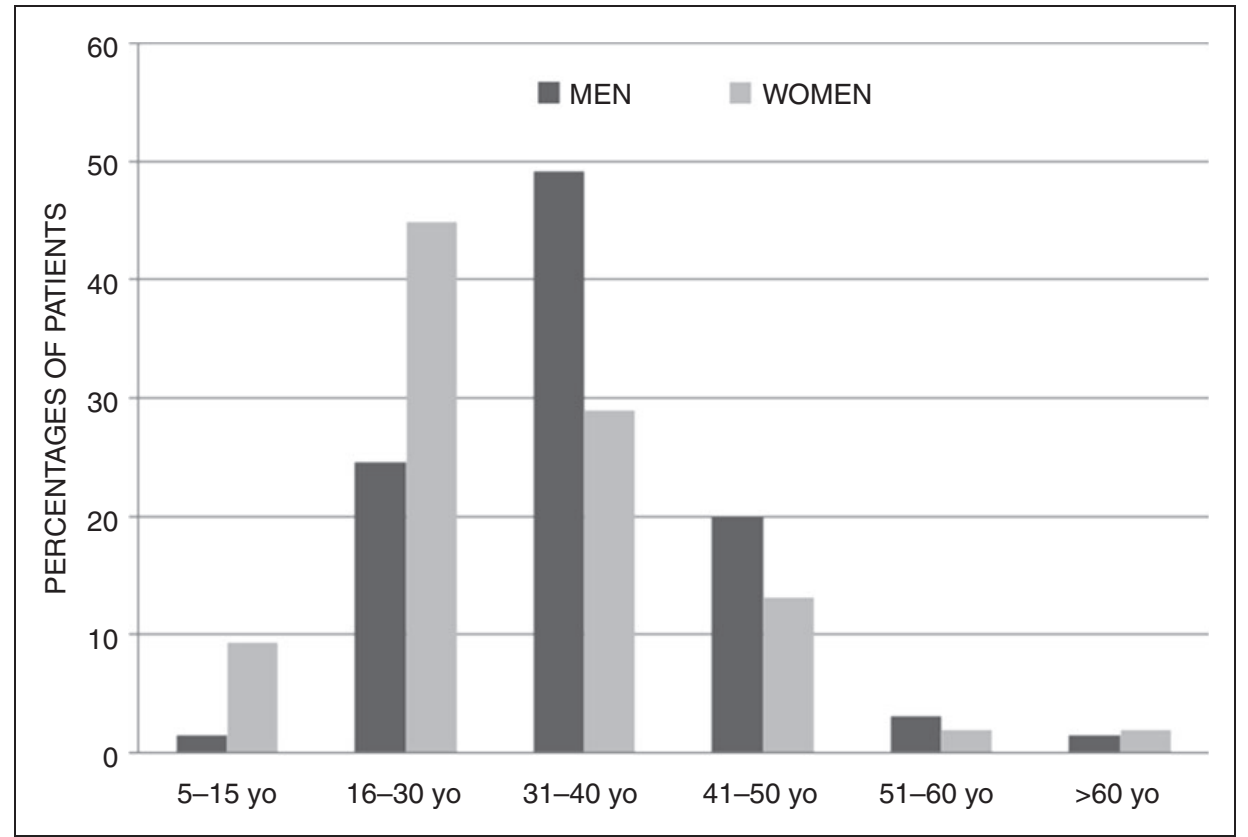

Figure 2. Age at onset of MA according to gender.

The peak age at onset of MA attacks is between 16 and 30 years in women and between 31 and 40 years in men. MA: migraine with aura; yo: years old. Missing data for one female individual.

The frequency of MA attacks during the two years before inclusion was found to vary widely among patients but was relatively low since $80.2 \%$ of patients reported less than one attack per month (Figure 1).

Among the 139 patients with MA who reported headache associated with aura, $87(65 \%)$ reported hemicranial pain during their MA attack, 89 (66.4\%) pulsatility, $99(74.5 \%)$ phonophobia and $98(73.7 \%)$ photophobia. In addition, $83(62.5 \%)$ complained of nausea and $57(43.2 \%)$ had vomiting during their headache. The mean duration of headache was between 4 and 72 hours in 71 individuals $(58.7 \%)$, and less 
than 4 hours in $37(30.6 \%)$. Only two participants with MA $(1.7 \%)$ reported headache episodes of more than 72 hours. For 11 patients $(9.1 \%)$, the mean duration of headache could not be assessed.

Common triggers of MA attacks such as stress, anxiety or fatigue were detected in 42 patients $(24.4 \%)$. Eight women reported menstrual MA attacks (corresponding to $7.4 \%$ of women with MA); only one had pure menstrual MA.

\section{Aura symptoms during MA attacks in the study population}

Visual symptoms during MA were reported by 141 patients ( $82 \%$ of patients with MA attacks), sensory symptoms occurred in 126 patients $(73.3 \%)$, aphasia in $96(55.8 \%)$ and motor symptoms in $35(20.3 \%)$. Scintillating scotoma was the most frequent visual symptom reported by $80(46.5 \%)$ patients with MA. Lateral homonymous hemianopia, phosphenes and blindness (visual field alteration preventing them from discerning the main characteristics of the environment) were reported respectively by $44(25.6 \%), 37(21.5 \%)$ and three $(1.7 \%)$ MA patients. Sensory symptoms involved the arm in 119 patients with MA $(69.2 \%)$, the face in $83(48.3 \%)$ and the leg in $47(27.3 \%)$. Speech disturbances during aphasic auras included alterations of oral expression in 66 patients $(38.4 \%)$, paraphasia in $30(17.4 \%)$, dysarthria in $18(10.5 \%)$ and impaired comprehension in seven $(4.1 \%)$. Motor symptoms were reported in the arm in 32 patients with MA $(18.6 \%)$, in the leg in $15(8.7 \%)$ or face in 10 $(5.8 \%)$. Thirty-seven patients $(21.5 \%$ of patients with MA) reported only one type of aura symptom. Twentytwo patients $(12.8 \%$ of MA patients) reported only visual aura, 12 patients (7\%) only sensitive aura, one patient $(0.6 \%)$ only aphasic aura and one patient $(0.6 \%)$ only confusion (c.f. below for description of patients with atypical auras).

A total of 119 patients (69.2\% of patients with MA) were able to report a typical 'marche migraineuse' with a progression of symptoms over $\geq 5$ minutes during their auras.

For 34 patients $(19.7 \%$ of patients with MA), the aura was never accompanied by headache.

Among the 173 patients with MA, $102(59.3 \%)$ individuals reported at least one atypical aura: 35 patients (20.3\% of MA patients) ever had motor symptoms during their aura; 35 others previously reported basilar or other unusual symptoms such as confusion, alterations of consciousness or hallucinations $(20.3 \%)$. Fifty-three patients reported acute-onset auras $(30.8 \%)$ and eight patients long-lasting auras $(4.7 \%)$. In the present cohort, none had a history of coma or meningism.

\section{MA as inaugural or isolated symptom of the disease}

MA was the inaugural manifestation of the disease in 147 patients, which represents $41.1 \%$ of symptomatic patients of our cohort. After exclusion of the 43 dementia patients in order to prevent a potential recall bias, MA remained the most frequent inaugural symptom of the disease (in $44.4 \%$ of the 315 symptomatic patients without dementia). Inaugural clinical manifestations of the disease distinct from MA are reported in the supplemental material.

MA was observed as the sole symptom of the disease (no history of stroke, epilepsy, dementia or psychiatric disorder except for a possible episode of depression) in 46 patients of the cohort $(12.1 \%)$. Their mean age was 43 years (range: $25-72$ years). In this subgroup of MA patients, 26 individuals $(56.5 \%)$ reported at least one episode of atypical aura (detailed in the supplemental material). All patients who presented MA as the sole symptom of CADASIL at the time of the study except one had WMH on MRI.

\section{Inter- and intra-familial variability of the migraine phenotype}

Data obtained from seven large multiplex families with four or more affected individuals included in the study are reported in Figure 3. Each family comprised at least two individuals with MA; the results showed that the age at onset of MA can largely differ among migraineurs within a same family, with a maximal difference of about 45 years (family 3 ). In addition, characteristics of aura symptoms and frequency of MA attacks also broadly vary among related migraineurs.

The number of mutations in the cohort was too high (71 different mutations were identified) for analyzing potential genotype-phenotype correlations in the present cohort.

\section{Demographic, clinical and imaging correlates of MA in the study population}

Patients who had a history of MA were significantly younger and comprised a larger proportion of women than patients without MA. After adjustment for age, gender, current cardiovascular risk factors and educational level, a positive history of MO was found significantly more frequently in patients with MA than in the rest of the sample. Slightly higher MMSE, MDRS and Initiation/Perseveration MDRS subscale scores and lower Rankin score were observed in the MA group despite adjustment for multiple confounders (Table 2). Similarly, better cognitive and functional performances were also found in all migraine patients 


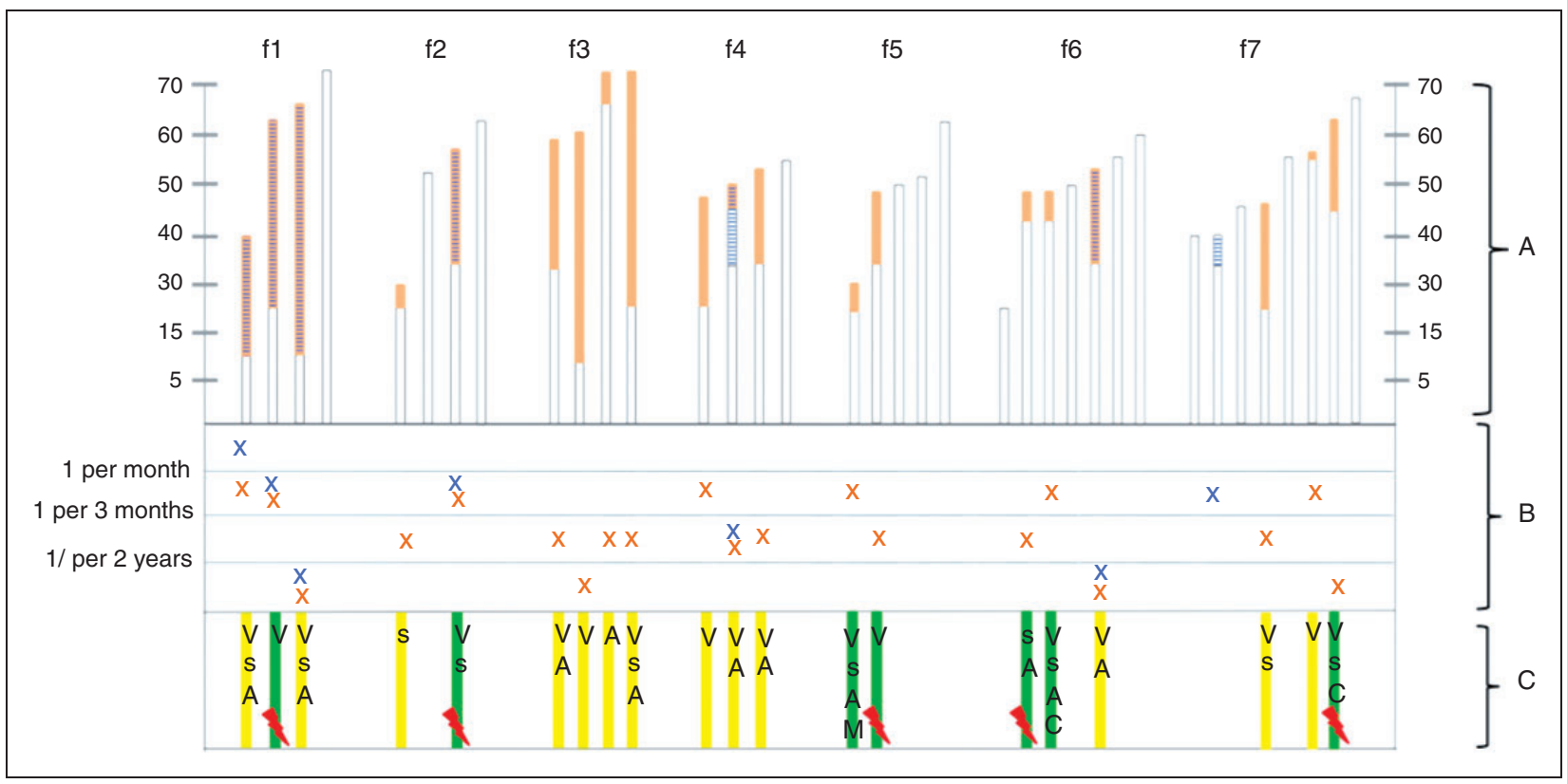

Figure 3. Migraine phenotype in seven French CADASIL families with more than four CADASIL patients in the cohort. (A) A vertical rod represents a patient and the height of the rod indicate his or her age at inclusion. Patients within a single family are grouped and families are numbered from $\mathrm{fl}$ to $\mathrm{f}$. The orange bands represent age at MA onset and the blue-hatched areas indicate age at MO onset.

(B) The red crosses indicate the mean frequency of MA attacks and the blue crosses indicate the mean frequency of $M O$ attacks during the two years before inclusion.

(C) This panel gives information about the MA symptoms. The yellow strips indicate patients having only typical auras whereas the green strips indicate patients reporting atypical auras. Symptoms are represented by a capital letter (V: Visual, S: sensory, A: aphasic, M: motor, C: confusion and/or decreased level of consciousness). Patients reporting acute-onset auras are specified with a red lightning symbol.

CADASIL: cerebral autosomal dominant arteriopathy with subcortical infarcts and leukoencephalopathy; MA: migraine with aura; MO: migraine without aura.

(MA and/or MO patients) in comparison with those without migraine (Table S1, supplemental material).

Regarding the MRI parameters, there was a trend for a lower mean number of microbleeds and a lower load of WMH in the MA group after adjustment for age, gender and cardiovascular risk factors (Table 2). The lower number of microbleeds was found to be significant when all migraine patients, including MA and/ or MO patients, were compared to the other individuals of the cohort (Table 1S, supplementary material).

\section{Discussion}

In this large cohort of CADASIL patients, the prevalence of migraine attacks exceeded $50 \%$ of individuals and appeared higher than previously reported in the literature $(4-6,13)$. The use of a structured interview for diagnosis and prospective recruitment of patients, obtained only in the present study, may explain this discrepancy. In addition, selection bias related to the centralization of recruitment in each country cannot be totally excluded. This high prevalence appears mainly related to the $45.8 \%$ prevalence of MA in the sample, which is nearly 10 times higher than that observed in the general population. The small difference in the prevalence of MA between men and women in CADASIL patients contrasting with the 1:2 male:female ratio in the general population further illustrates the specific consequences of the genetic disease. In contrast, MO attacks are reported only by $23 \%$ of individuals in the cohort. This frequency is two times lower than the frequency of MA in the sample and appears only slightly higher than the prevalence of $\mathrm{MO}$ in the general population, varying from $6 \%$ to $18 \%(7,8,14,15)$. MO attacks were also detected in $18 \%$ of men and $28 \%$ of women, which is close to the sex ratio of MO in the general population (8). Finally, MO attacks were reported two times more frequently in CADASIL patients with MA than in those without MA which suggests that the predisposition for MA in CADASIL may favor, but only moderately, the co-occurrence of MO attacks.

There is accumulating evidence supporting a causal relationship between cortical spreading depression 
Table 2. Differences observed between patients with MA and patients without MA.

\begin{tabular}{|c|c|c|c|c|}
\hline & \multicolumn{2}{|l|}{ Migraine with aura } & \multirow[b]{2}{*}{$p^{\mathrm{a}}$} & \multirow[b]{2}{*}{$p^{\mathrm{b}}$} \\
\hline & Yes $(n=173)$ & No $(n=205)$ & & \\
\hline Mean age (range) & $47.8(25-72)$ & $54.2(23-79)$ & $<0.0001$ & \\
\hline Women & $62.4 \%$ & $50.2 \%$ & 0.018 & $<0.0001$ \\
\hline \multicolumn{5}{|l|}{ Cardiovascular risks factors } \\
\hline Alcohol consumption $>2$ drinks per day ${ }^{c}$ & $5.3 \%$ & $9.9 \%$ & 0.095 & \\
\hline Current or past smokers & $52.6 \%$ & $54.6 \%$ & 0.693 & \\
\hline Arterial hypertension & $17.3 \%$ & $25.5 \%$ & 0.056 & \\
\hline Diabetes & $1.2 \%$ & $3.9 \%$ & 0.117 & \\
\hline Hypercholesterolemia & $33.1 \%$ & $47.8 \%$ & 0.004 & \\
\hline \multicolumn{5}{|l|}{ Associated neurological signs } \\
\hline $\mathrm{MO}$ & $31.2 \%$ & $16.1 \%$ & 0.0005 & 0.01 \\
\hline Stroke & $53.8 \%$ & $68.8 \%$ & 0.0027 & 0.23 \\
\hline Psychiatric disorder & $40.5 \%$ & $45.9 \%$ & 0.29 & 0.38 \\
\hline Epilepsy & $6.4 \%$ & $11.7 \%$ & 0.077 & 0.30 \\
\hline Dementia & $6.9 \%$ & $15.2 \%$ & 0.012 & 0.25 \\
\hline Mean MMSE (SD) & $27.97(3.0)$ & $25.59(5.3)$ & $<0.0001$ & 0.0015 \\
\hline Mean Rankin (SD) & $0.66(1.1)$ & $\mathrm{I} .40(\mathrm{I} .5)$ & $<0.0001$ & 0.002 \\
\hline Mean MDRS (SD) & I37.7 (I2.3) & $129.6(21.3)$ & $<0.0001$ & 0.0143 \\
\hline Mean I/P MDRS subscale (SD) & $34.9(4.6)$ & $31.3(8.3)$ & $<0.0001$ & 0.007 \\
\hline \multicolumn{5}{|l|}{ MRI parameters } \\
\hline WMH volume, mean (SD) & $0.068 \mathrm{I}(0.05)$ & $0.0734(0.05)$ & 0.318 & 0.07 \\
\hline Lacunar volume, mean (SD) & $2.59 .10^{-4}\left(5.3 .10^{-4}\right)$ & $2.61 .10^{-4}\left(4 \cdot 5 \cdot 10^{-4}\right)$ & 0.96 & 0.28 \\
\hline Microbleeds, mean (SD) & $1.29(4.3)$ & $5.91(17.3)$ & $<0.0001$ & 0.05 \\
\hline BPF, mean (SD) & $86.46(5.3)$ & $84.24(7.2)$ & 0.003 & 0.48 \\
\hline
\end{tabular}

(a) $p$ value without adjustment. (b) $p$ value obtained after adjustment for age, gender, cardiovascular risk factors, educational level. (c) $>2$ drinks per day for men and $>$ I drink per day for women.

BPF: brain parenchymal fraction; MDRS: Mattis Dementia Rating Scale; I/P MDRS: Initiation/Perseveration MDRS; MMSE: Mini-Mental State Examination; SD: standard deviation; WMH: white matter hyperintensities; MO: migraine without aura.

(CSD) and aura symptoms. Different factors were found capable of modulating the threshold for triggering CSD in experimental data (16-19). In transgenic mice over-expressing NOTCH3 mutations, Eikermann-Haerter et al. detected an enhanced susceptibility to CSD (20). The exact origin of this reduced threshold for CSD remains unknown. In the CADASIL mouse model, this is observed in the absence of major reduction of cortical blood flow. In humans, our results confirm that MA is mostly reported at the earliest stage of the disease and was even the inaugural manifestation in $41 \%$ of individuals. Thus, aura symptoms usually occur when ischemic manifestations of the disease have not yet begun. Aura symptoms can also occur in the absence of MRI lesions in CADASIL (21) as observed in a single individual of our cohort. In addition, our results also suggest that the frequency of MA attacks decreases at the advanced stage of the disease when both ischemic lesions and manifestations are increasing. Other studies are needed to determine whether changes occurring at the cortex level in myelin content or in microglial or neuronal activity during the progression of the disease are involved $(22,23)$. Altogether, these data further support that cerebral ischemia is not the primary mechanism of aura symptoms in CADASIL and that mutations of the NOTCH3 gene are actually responsible for early changes in cortical excitability in humans. Otherwise, the prevalence of tension typeheadache (TTH) appears to be quite low in our cohort in comparison to its prevalence in the general population. However, we cannot exclude that TTH has been masked by the frequent occurrence of migraine headache in our patients, or by the use of antalgic treatments, thus leading to an underestimation of prevalence of TTH in our CADASIL cohort.

In the present study, we found a significant female preponderance among CADASIL patients with MA. Age of onset of MA was also significantly lower in women than in men. These results are in line with 
previous findings in CADASIL $(9,24)$. They are also in agreement with the female preponderance of migraineurs in the general population, although more pronounced for MO than for MA $(7,14,25,26)$. Our data suggest that, in addition to the genetic defect, the hormonal status may continue to influence the occurrence of MA in CADASIL patients. Female hormones have been repeatedly shown to facilitate CSD in different animal models $(18,19,27)$. In transgenic mice with NOTCH3 mutations, females were also found to have a lower CSD trigger threshold than males although the difference did not reach statistical significance (20). Some authors previously raised the hypothesis that MO could also result from silent waves of CSD $(28,29)$. The disproportionate increase of MA attacks compared to MO attacks in CADASIL do not favor this interpretation. We also observed that the age at onset, characteristics of aura symptoms and frequency of attacks vary broadly among migraineurs within the same family, which suggests that the genetic familial background may not have a significant influence on the development of MA symptoms in CADASIL.

Almost $60 \%$ of individuals with MA in the cohort already experienced at least one atypical aura consisting of unusual symptoms such as motor deficit, loss of consciousness, fever, acute onset or long-lasting aura. The high incidence of atypical MA attacks has already been reported (9) and may raise important diagnostic difficulties. Particularly, acute-onset aura was detected in one-third of MA patients in this cohort and may be difficult to discriminate from TIAs. The frequent occurrence of "positive" neurologic symptoms such as paresthesia or visual scintillations or the stereotyped nature of repetitive attacks are then particularly helpful for their recognition. Moreover, when the nature of a symptom was uncertain, the episode was classified as an episode indistinguishable between aura and TIA in the present study, thus reducing the risk of overestimation of aura symptoms. In the absence of other manifestations of the disease, the occurrence of atypical aura may also be useful for suggesting the diagnosis of CADASIL, particularly in at-risk individuals or in the presence of MRI signal abnormalities. In our patients with MA who had a normal neurological examination and no other symptom of the disease except possible depression, atypical aura was detected in more than half of individuals.

In our cohort, we observed that patients with MA had globally a better functional and cognitive outcome than the other individuals. After adjustment for multiple confounding factors, patients with MA had higher MMSE and MDRS scores and lower Rankin scores than the other participants. These results further support that the occurrence of MA is not associated with the development of ischemic lesions and disability in CADASIL and may even present some protective effects. There are experimental data showing that CSD may induce long-lasting brain protection against the development of ischemic lesions $(30,31)$. We think however that these findings should be interpreted with caution. Particularly, some recall biases cannot be totally excluded. For instance, patients with alterations in their memory performances are less likely to report their past history of migraine attack in comparison to patients with preserved cognitive functions. In addition, the frequent use of aspirin in patients who had a stroke may influence the occurrence of MA attacks and consequently their recognition. More, we note a trend toward a lower proportion of stroke in the group of patients with MA, but this result should be interpreted with caution because episodes "indistinguishable between aura and TIA" have been classified as stroke, and this could have introduce a bias due to a misclassification of such episodes.

\section{Clinical implications}

- Migraine with aura, observed in almost half of all cerebral autosomal dominant arteriopathy with subcortical infarcts and leukoencephalopathy (CADASIL) patients, is the most frequent inaugural symptom of the disease and can remain isolated.

- The occurrence of atypical aura should evoke the diagnosis of CADASIL, particularly in the presence of white-matter lesions or in a suggestive familial context, since almost $60 \%$ of migraine CADASIL patients report atypical aura.

- Migraine with aura does not appear to be associated with a negative prognosis in CADASIL.

\section{Acknowledgements}

The authors acknowledge all patients who participated in this research, their families and the association CADASIL France for their active collaboration; Jocelyne Ruffié and Solange Hello for their involvement in the practical organization of the study; Marie Germaine Bousser and Annie Kurtz for their advice and constant support during the study; and the Unité de Recherche Clinique of Saint-Louis/Lariboisiere Hospital, Paris France (Véronique Jouis, Laurence Guery) for their technical support. 


\section{Declaration of conflicting interests}

The authors declared no potential conflicts of interest with respect to the research, authorship, and/or publication of this article.

\section{Funding}

The authors disclosed receipt of the following financial support for the research, authorship, and/or publication of this article: This study is supported by grants from the French Ministry of Health (Regional and National PHRC AOR 02-001), ARNEVA (Association de Recherche en NEurologie Vasculaire), the Vascular Dementia Research Foundation, and the Fondation Leducq (Transatlantic Network of Excellence on the Pathogenesis of Small Vessel Disease of the Brain) (http://fondationleducq.org).

\section{References}

1. Joutel A, Corpechot C, Ducros A, et al. Notch3 mutations in CADASIL, a hereditary adult-onset condition causing stroke and dementia. Nature 1996; 383: 707-710.

2. Joutel A, Vahedi K, Corpechot C, et al. Strong clustering and stereotyped nature of Notch3 mutations in CADASIL patients. Lancet 1997; 350: 1511-1515.

3. Chabriat H, Joutel A, Dichgans M, et al. Cadasil. Lancet Neurol 2009; 8: 643-653.

4. Chabriat H, Vahedi K, Iba-Zizen MT, et al. Clinical spectrum of CADASIL: A study of 7 families. Cerebral autosomal dominant arteriopathy with subcortical infarcts and leukoencephalopathy. Lancet 1995; 346: 934-939.

5. Dichgans M. The phenotypic spectrum of CADASIL: Clinical findings in 102 cases. Ann Neurol 1998; 44: 731-739.

6. Desmond DW, Moroney JT, Lynch T, et al. The natural history of CADASIL: A pooled analysis of previously published cases. Stroke 1999; 30: 1230-1233.

7. Rasmussen BK and Olesen J. Migraine with aura and migraine without aura: An epidemiological study. Cephalalgia 1992; 12: 221-228; discussion 186.

8. Russell MB, Rasmussen BK, Fenger K, et al. Migraine without aura and migraine with aura are distinct clinical entities: A study of four hundred and eighty-four male and female migraineurs from the general population. Cephalalgia 1996; 16: 239-245.

9. Vahedi K, Chabriat H, Levy C, et al. Migraine with aura and brain magnetic resonance imaging abnormalities in patients with CADASIL. Arch Neurol 2004; 61: $1237-1240$.

10. International Headache Society. The International Classification of Headache Disorders, 2nd edition. Cephalalgia 2004; 24(Suppl 1): 16-22.

11. Viswanathan A, Guichard JP, Gschwendtner A, et al. Blood pressure and haemoglobin A1c are associated with microhaemorrhage in CADASIL: A two-centre cohort study. Brain 2006; 129: 2375-2383.

12. Durand-Dubief F, Belaroussi B, Armspach JP, et al. Reliability of longitudinal brain volume loss measurements between 2 sites in patients with multiple sclerosis: Comparison of 7 quantification techniques. AJNR Am J Neuroradiol 2012; 33: 1918-1924.

13. Liem MK, Oberstein SAJL, van der Grond J, et al. CADASIL and migraine: A narrative review. Cephalalgia 2010; 30: 1284-1289.

14. Lipton RB and Bigal ME. The epidemiology of migraine. Am J Med 2005; 118(Suppl 1): 3S-10S.

15. Rasmussen BK and Olesen J. Epidemiology of migraine and tension-type headache. Curr Opin Neurol 1994; 7: 264-271.

16. Lauritzen M. Pathophysiology of the migraine aura. The spreading depression theory. Brain 1994; 117(Pt 1): 199-210.

17. Lauritzen M, Dreier JP, Fabricius M, et al. Clinical relevance of cortical spreading depression in neurological disorders: Migraine, malignant stroke, subarachnoid and intracranial hemorrhage, and traumatic brain injury. J Cereb Blood Flow Metab 2011; 31: 17-35.

18. Eikermann-Haerter K, Dileköz E, Kudo C, et al. Genetic and hormonal factors modulate spreading depression and transient hemiparesis in mouse models of familial hemiplegic migraine type 1. J Clin Invest 2009; 119: 99-109.

19. Eikermann-Haerter K, Kudo C and Moskowitz MA. Cortical spreading depression and estrogen. Headache 2007; 47(Suppl 2): S79-S85.

20. Eikermann-Haerter K, Yuzawa I, Dileköz E, et al. Cerebral autosomal dominant arteriopathy with subcortical infarcts and leukoencephalopathy syndrome mutations increase susceptibility to spreading depression. Ann Neurol 2011; 69: 413-418.

21. Monteiro C, Barros J, Taipa R, et al. Sporadic hemiplegic migraine with normal imaging as the initial manifestation of CADASIL. Cephalalgia 2012; 32: 255-257.

22. Merkler D, Klinker F, Jürgens T, et al. Propagation of spreading depression inversely correlates with cortical myelin content. Ann Neurol 2009; 66: 355-365.

23. Pusic KM, Pusic AD, Kemme J, et al. Spreading depression requires microglia and is decreased by their M2a polarization from environmental enrichment. Glia 2014; 62: 1176-1194.

24. Gunda B, Hervé D, Godin O, et al. Effects of gender on the phenotype of CADASIL. Stroke 2012; 43: 137-141.

25. Launer LJ, Terwindt GM and Ferrari MD. The prevalence and characteristics of migraine in a populationbased cohort: The GEM study. Neurology 1999; 53: 537-542.

26. Russell MB, Rasmussen BK, Thorvaldsen P, et al. Prevalence and sex-ratio of the subtypes of migraine. Int $J$ Epidemiol 1995; 24: 612-618.

27. Brennan KC, Romero Reyes M, López Valdés HE, et al. Reduced threshold for cortical spreading depression in female mice. Ann Neurol 2007; 61: 603-606.

28. Cao Y, Welch KM, Aurora S, et al. Functional MRIBOLD of visually triggered headache in patients with migraine. Arch Neurol 1999; 56: 548-554.

29. Woods RP, Iacoboni M and Mazziotta JC. Brief report: Bilateral spreading cerebral hypoperfusion during spontaneous migraine headache. $N$ Engl J Med 1994; 331: 1689-1692. 
30. Matsushima K, Schmidt-Kastner R, Hogan MJ, et al. Cortical spreading depression activates trophic factor expression in neurons and astrocytes and protects against subsequent focal brain ischemia. Brain Res 1998; 807: 47-60.
31. Yanamoto H, Xue JH, Miyamoto S, et al. Spreading depression induces long-lasting brain protection against infarcted lesion development via $B D N F$ gene-dependent mechanism. Brain Res 2004; 1019: 178-188. 